\title{
Characterization of vortex shedding on a hydrofoil using PIV measurements
}

\author{
H. Bonnard ${ }^{1 *}$, L. Chatellier ${ }^{1}$, L. David ${ }^{1}$ \\ ${ }^{1}$ Institut P', UPR 3346 CNRS - Université de Poitiers - ISAE-ENSMA, France \\ *herve.bonnard@univ-poitiers.fr
}

\begin{abstract}
An experimental study of vortex shedding on a hydrofoil Eppler 817 was conducted using two-dimensional two components Particle Image Velocimetry. This foil section's characteristics are adapted for naval applications but sparsely documented. The characterization of the flow modes was realized based on statistical data such as the mean velocity field and the standard deviation of the vertical velocities. The data were acquired at very low Reynolds number which are not often covered for such hydrofoil and at four angles of attack ranging from $2^{\circ}$ to $30^{\circ}$. A map of different characteristic flow modes was made for this space of parameters and was used to identify flow configurations exhibiting particular dynamics.
\end{abstract}

\section{Introduction}

While lifting surfaces are already everywhere on ships, from the rudder to the propeller, the last decades have seen an important rise on the uses of hydrofoils, which goal is to lift a boat out of the water just as a plane's wing. The sailing world has witnessed the high potential of those hydrofoils, as shown by their increasing use in major sailing events such as the America's cup or the Vendée Globe. The sections of hydrofoils can be quite different from airfoils as they have to be designed with the specific operating conditions of the sea, such as the presence of the free-surface and the risk of cavitation. The latter can be controlled by designing specific hydrofoils shapes which minimize the pressure loss on the suction side. Such profile was for example designed by Eppler (1990) and Eppler and Shen (1979) by prescribing a velocity distribution at different angle of attack and using an inverse method based on a combination of a panel method to analyse a potential flow with a boundary-layer method.

The flow characteristic modes of an Eppler 817 (E817) hydrofoil were studied experimentally using Particles Image Velocimetry (PIV) measurements over a range of low chord-based Reynolds numbers 0.4 . $10^{3} \leq R e_{c} \leq 20 \cdot 10^{3}$ and four angles of attack $\alpha=2^{\circ}, 6^{\circ}, 12^{\circ}, 30^{\circ}$.

The PIV measurements were done on a very large field (about 3.5 chords upstream and downstream of the hydrofoil) even at the cost of a coarser resolution, in order to analyse the whole flow, including a region of the flow with little influence of the hydrofoil. Those data are especially useful for an ongoing project on data assimilation for which knowing the upstream conditions is crucial to define the simulation's inlet. Meldi and Poux (2017) and Suzuki et al. (2018) highlighted the difficulties of assimilating experimental data due to the limited extent of the physical domain covered by the PIV data as well as the under-sampling of experimental data compared to the time steps of numerical simulations. The data acquired with this study will allow us to investigate the effect of these parameters on different flows topologies and dynamics.

\section{Experimental setup}

The experiments were carried out at the P' Institute on the Environmental Hydrodynamic Platform's open water channel which dimensions are $7 \times 0.385 \times 0.6 \mathrm{~m}(\mathrm{LxWxH})$. The channel is equipped with a PCM Moineau worm-drive pump controlled with a Schneider Electric variable-frequency drive up to flow rates of $Q=65 \mathrm{~L} / \mathrm{s}$. The flow rates are measured with an Endress+Hauser Promag55 electromagnetic flowmeter. The free-surface height is modified by a spillway gate and was set to $h=280 \mathrm{~mm}$ for the measures. The hydrofoil (chord $c=40 \mathrm{~mm}$ and span $s=355 \mathrm{~mm}$ ) was placed in the middle of the water volume such as the 
distance between the profile and either the free-surface or the bottom wall was 3.5 chords. The experimental setup is illustrated on Figure 1.

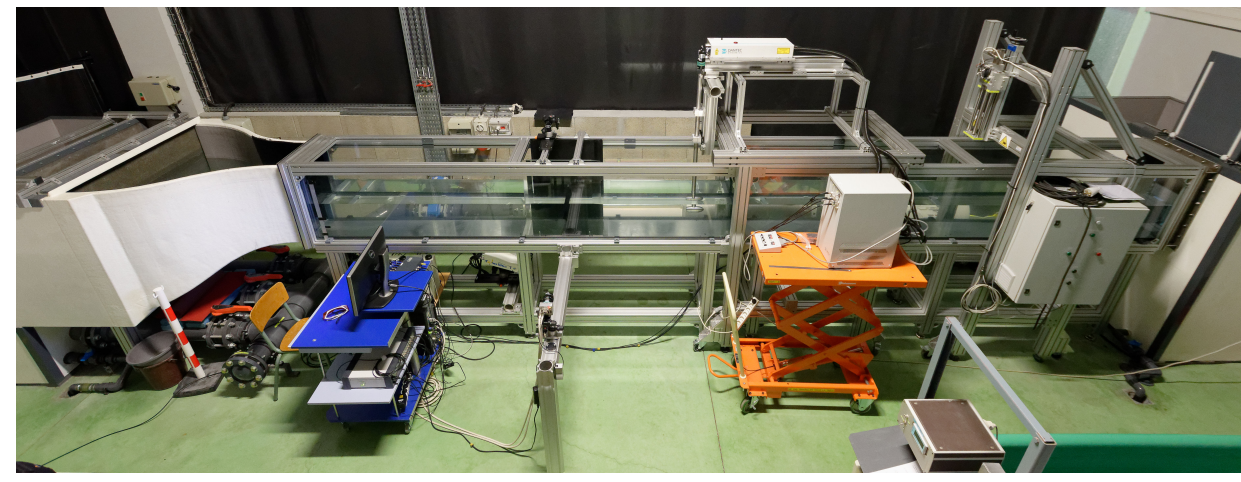

Figure 1: Overview of the water channel with wing model support and PIV setup.

Preliminary flow visualizations were performed using a $1 \mathrm{~W}$ continuous laser diode in order to describe the main flow characteristics. Long exposure images were taken in order to get a representation of the flow topology through the particles' trajectories. Four characteristics flow and vortex shedding regimes were observed: attached flow, trailing-edge vortex, separation bubble and leading-edge vortex (see Yarusevych et al. (2009) Huang et al. (2001)). The transition between the first two modes can be observed in Figure 2 .

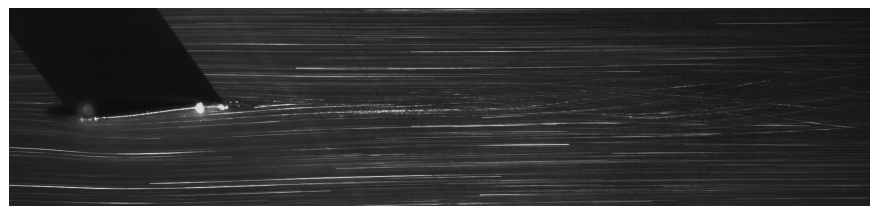

(a) $R e_{c}=4700$, attached-flow

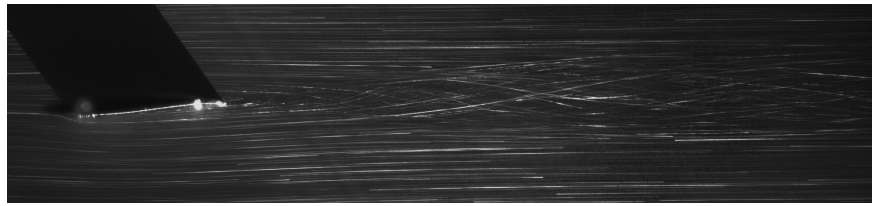

(b) $R e_{c}=5200$, trailing-edge vortices

Figure 2: Comparison of the flow characteristic regimes at $\alpha=2^{\circ}$ for two close $\operatorname{Re}_{c}$

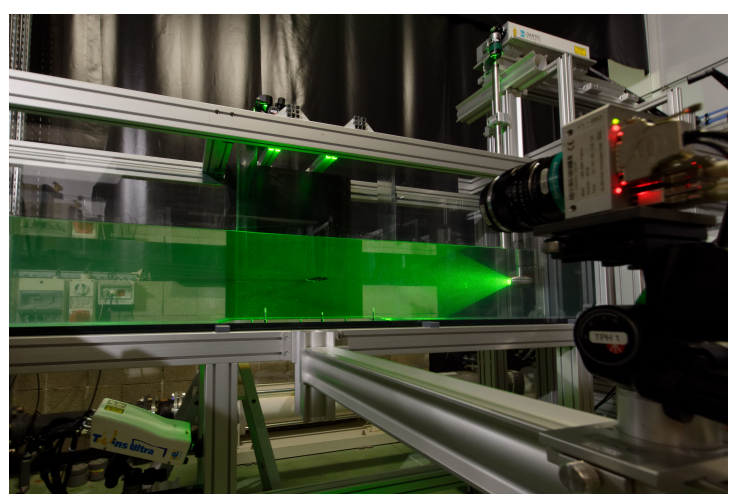

Figure 3: Photograph of the setup with the laser sheet using the underwater illumination unit

PIV measurements were carried out using a Dantec SpeedSense 10404 MP $100 \mathrm{~Hz}$ camera on 16 cases (4 Reynolds numbers and 4 angles of attack) chosen based on the previous observations. As an opaque $3 \mathrm{D}$-printed ABS hydrofoil was used, two lasers were required in order to get the largest illumination. A $2 \mathrm{x}$ $30 \mathrm{~mJ}$ Nd:YAG Quantel Twins Ultra $(532 \mathrm{~nm}, 20 \mathrm{~Hz})$ was placed below the water channel to illuminate the pressure side of the foil as well as the upstream flow. As the free-surface of the open water tunnel prevent the use of a laser from the top of the experiment, a 2 x $50 \mathrm{~mJ}$ Nd:YAG Litron Nano L 50-50 PIV (532 nm, $50 \mathrm{~Hz}$ ) was used with LaVision underwater illumination unit in order to view the suction side of the foil and the downstream flow. The measurement equipments are depicted on Figure 3 .

A first series of measurements was performed on the 16 cases using 1000 double-frame images acquired at a very low frequency $(2$ to $5 \mathrm{~Hz}$ ) in order to provide statistically relevant datasets. Then, using only the fastest of the two lasers, Time-Resolved PIV (TR-PIV) series of 10000 images were acquired for the two lowest Reynolds numbers at two angles of attack $\left(\alpha=2^{\circ}\right.$ and $\left.12^{\circ}\right)$. A multi-frame PIV method was applied to evaluate the time resolved flow fields using a Fluid Trajectory Evaluation based on an Ensemble-averaged cross-correlation (FTEE) technique, see Jeon et al. (2014). Those two datasets provide an understanding of both the global mean flow and the dynamics of the flow fluctuations. 

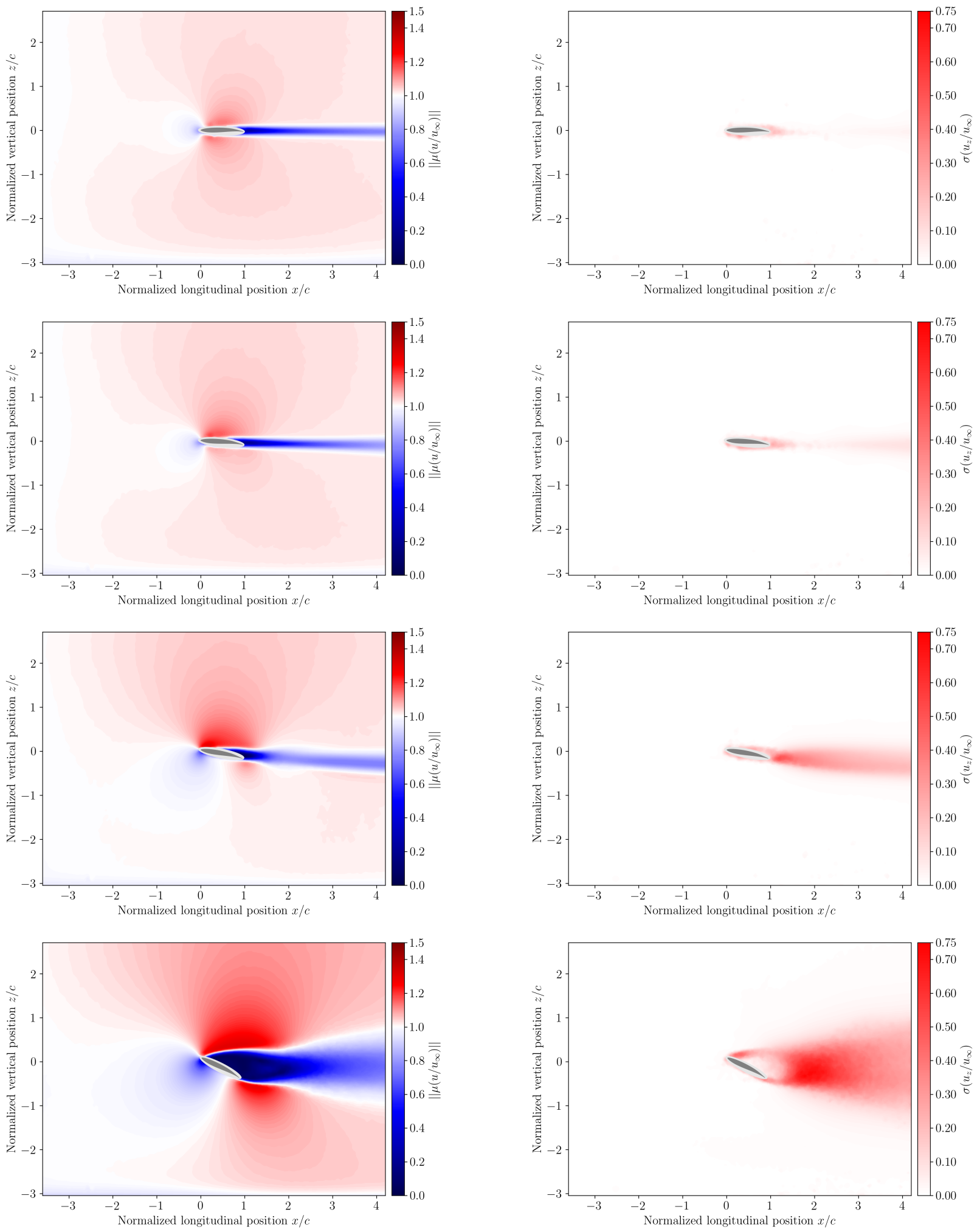

Figure 4: Mean normalized velocities (left) and standard deviation of normalized vertical velocities (right) at $R e_{c}=1400$ for $\alpha=2^{\circ}, 6^{\circ}, 12^{\circ}, 30^{\circ}$ (rows). The hydrofoil is represented as a dark grey area and the light grey mask corresponds to a zone where data could not be obtained accurately. 

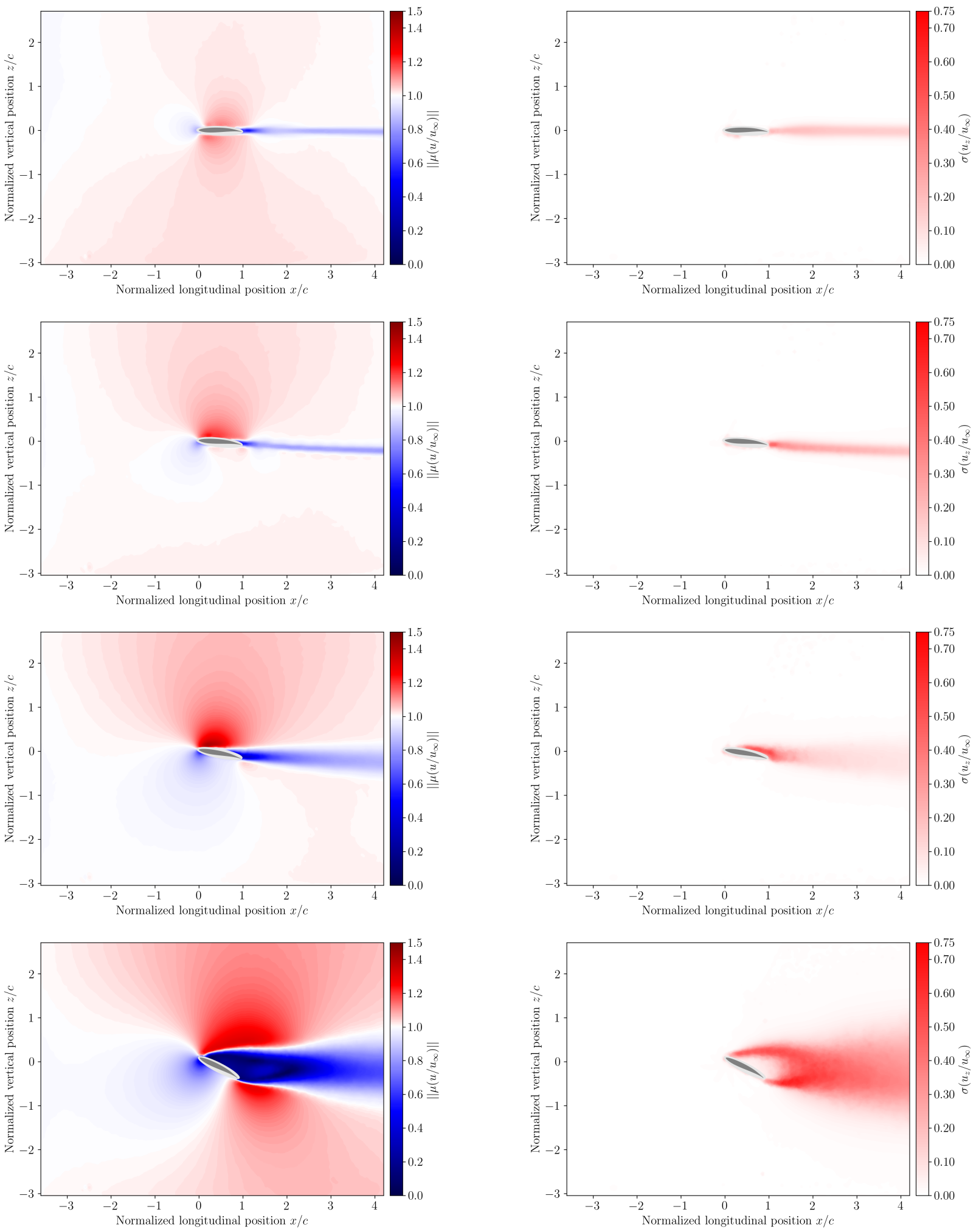

Figure 5: Mean normalized velocities (left) and standard deviation of normalized vertical velocities (right) at $R e_{c}=6300$ for $\alpha=2^{\circ}, 6^{\circ}, 12^{\circ}, 30^{\circ}$ (rows). The hydrofoil is represented as a dark grey area and the light grey mask corresponds to a zone where data could not be obtained accurately. 


\section{Results}

The mean normalized velocity field as well as the standard deviation of the normalized vertical velocities are represented on figures 4 and 5 for two of the Reynolds numbers studied at all the angle of attack. They both allow to see the time averaged topology of the flow around the hydrofoil at different conditions and can be used to identify the different flow modes.

For both chord base Reynolds number, at the lowest angle of attack $\left(\alpha=2^{\circ}\right)$ we can see an almost symmetrical velocity distribution, and thus pressure distribution, around the hydrofoil. This is due to the asymmetrical section of the Eppler 817, which has a negative lift coefficient at an angle of attack of $0^{\circ}$ and is at zero lift for an angle of attack of approximately $1.5^{\circ}$.

At $R e_{c}=1400$, when increasing the angle of attack from $2^{\circ}$ to $12^{\circ}$, the characteristic flow modes evolve from attached flow to a transitionary state and then a fully developed trailing edge vortex shedding. This can be seen using the standard deviation of the vertical velocity which increases in the wake as the angle of attack increases. While at $R e_{c}=6300$, we can see that the trailing edge vortex shedding start from the lowest angle of attack, reinforcing the preliminary results obtained with the laser diode showed in Figure 2. This is also illustrated by the mean velocity field where the wake is thinner than in the attached flow case from the lower Reynolds number.

At $R e_{c}=6300$ and $\alpha=12^{\circ}$, we observed a new mode: a separation vortex, which did not exist at this angle of attack for the lower Reynolds numbers. The standard deviation graph shows that the biggest fluctuations are closer to the hydrofoil, starting from about the third of the chord, which is characteristic of a separation. By comparing the mean velocity fields of the separated cased with the one at $R e_{c}=1400$ where it did not occur yet, we can see that the wake thicken progressively showing a triangular shape.

For all the Reynolds numbers studied, there is leading edge vortex shedding at the most extreme angle of attack investigated, $\alpha=30^{\circ}$. This was expected as the angle chosen is quite high.

The Figure 6 shows a cartography of the different vortex shedding types observed for all the cases studied. A zoom of the standard deviation of the vertical velocity graphs was added at some points to illustrate the differences between the modes. As there are very few data on this hydrofoil, especially at such low Reynolds numbers, our results can be compared, qualitatively, to the ones obtained on a well documented profile such as the NACA 0012 (see Huang et al. (2001)). Most patterns are similar with our study, such as the fact that trailing edge vortices start to appear at lower angle of attack when the Reynolds number increases. However, the separation vortices appeared at higher angles of attack, which was partly expected as our zero-lift angle of attack is not zero.

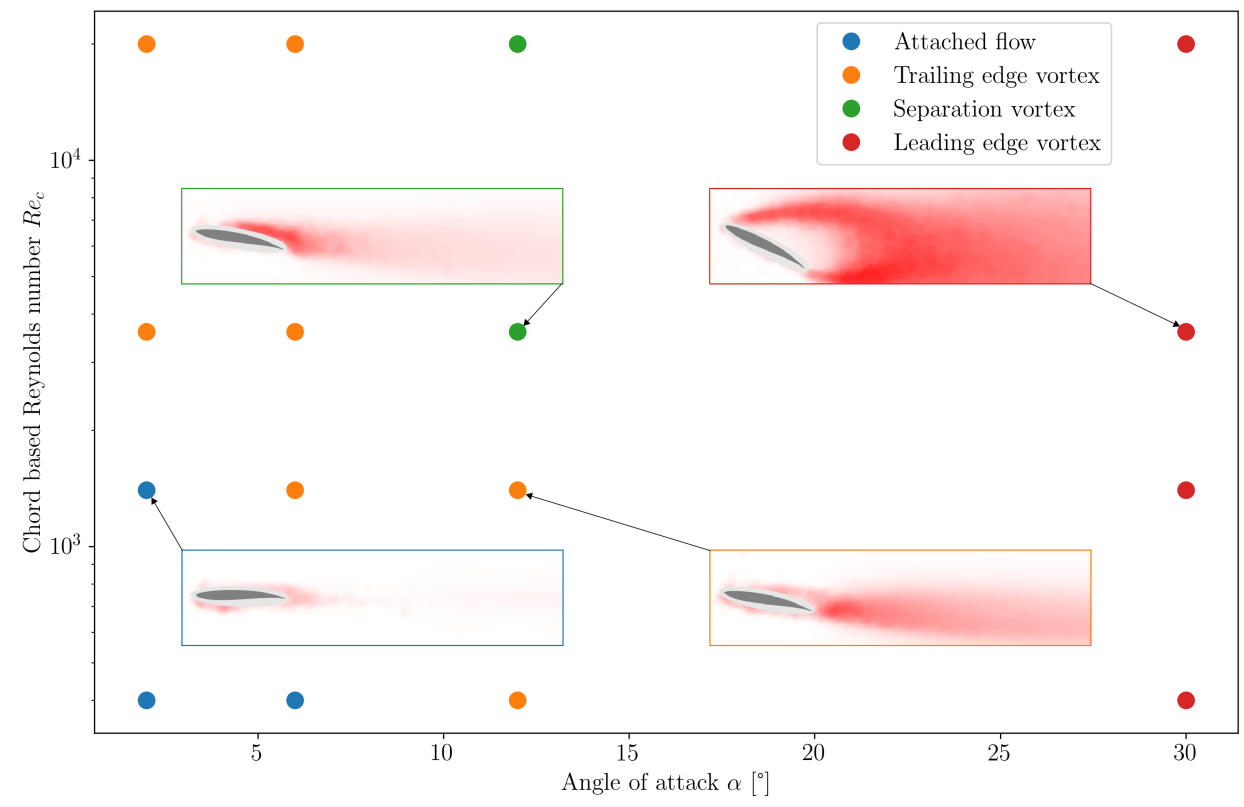

Figure 6: Flows modes observed on our Eppler 817 hydrofoil as a function of chord based Reynolds number $\operatorname{Re}_{c}$ and angle of attack $\alpha$. 


\section{Conclusions and perspectives}

The present study allowed us to map the different flow topologies around an Eppler 817 hydrofoil depending on the Reynolds number and angle of attack. This provided a better understanding of the flow characteristics around our hydrofoil and guided us when choosing interesting cases which will be further investigated.

A following experiment with a 3D T-shaped hydrofoil, that is a vertical part such as a rudder and a horizontal part at the end to produce lift, will be carried out on the same water channel. We will observe the evolution in behaviour when going from the extruded 2D section to a full 3D shape and the influence of the proximity with the free-surface trough various hydrofoil's depths.

\section{Acknowledgements}

The authors would like to thank the Direction Générale de l'Armement for the funding of Hervé Bonnard's $\mathrm{PhD}$ thesis as well as the European Research Council (ERC) for funding the project HOMER : Holistic Optical Metrology for Aero-Elastic Research under the European Union's Horizon 2020 research and innovation programme (grant agreement No 648161).

\section{References}

Eppler R (1990) Airfoil Design and Data. Springer Berlin Heidelberg, Berlin, Heidelberg

Eppler R and Shen YT (1979) Wing Sections for Hydrofoils-Part 1: Symmetrical Profiles. Journal of Ship Research

Huang RF, Wu JY, Jeng JH, and Chen RC (2001) Surface flow and vortex shedding of an impulsively started wing. Journal of Fluid Mechanics

Jeon YJ, Chatellier L, and David L (2014) Fluid trajectory evaluation based on an ensemble-averaged crosscorrelation in time-resolved PIV. Experiments in Fluids

Meldi M and Poux A (2017) A reduced order model based on Kalman filtering for sequential data assimilation of turbulent flows. Journal of Computational Physics

Suzuki T, Chatellier L, Jeon YJ, and David L (2018) Unsteady pressure estimation and compensation capabilities of the hybrid simulation combining PIV and DNS. Measurement Science and Technology

Yarusevych S, Sullivan PE, and Kawall JG (2009) On vortex shedding from an airfoil in low-Reynoldsnumber flows. Journal of Fluid Mechanics 